\title{
Phytochemical Screening and in Vitro Antimicrobial and Anticancer Activities of Different Extracts of Rosmarinus officinalis (Rosemary): A Comparative Study
}

\author{
Nada M. Ali \\ Department of Chemistry, College of Science, Albaha University, Albaha-1988, Saudi Arabia
}

Received March 29, 2021; Revised May 24, 2021; Accepted June 15, 2021

\begin{abstract}
Cite This Paper in the following Citation Styles
(a): [1] Nada M. Ali, "Phytochemical Screening and in Vitro Antimicrobial and Anticancer Activities of Different Extracts of Rosmarinus officinalis (Rosemary): A Comparative Study," Advances in Pharmacology and Pharmacy, Vol. 9, No. 3, pp. 44 - 55, 2021. DOI: 10.13189/app.2021.090302.
\end{abstract}

(b): Nada M. Ali (2021). Phytochemical Screening and in Vitro Antimicrobial and Anticancer Activities of Different Extracts of Rosmarinus officinalis (Rosemary): A Comparative Study. Advances in Pharmacology and Pharmacy, 9(3), 44 - 55. DOI: 10.13189/app.2021.090302.

Copyright $\bigcirc 2021$ by authors, all rights reserved. Authors agree that this article remains permanently open access under the terms of the Creative Commons Attribution License 4.0 International License

\begin{abstract}
Various medicinal plants are found in the dense forest of Albaha region, southwest of Saudi Arabia. These plant species are natively utilized for the prevention and treatment of various diseases. This study was designed to analyze the chemical composition of ethanolic, petroleum ether, chloroform, and methanolic extracts of Rosmarinus officinalis (rosemary) collected from Albaha region and evaluate the antimicrobial and cytotoxic activities of these extracts. Fresh aerial parts of $R$. officinalis (stem and leaves) were used for extraction. Then the crude extracts were investigated by using gas chromatography- mass spectrometry (GC-MS) technique to determine their chemical constituents. Antimicrobial assays were performed using Bacillus subtilis and Staphylococcus aureus (Gram-positive bacteria), Escherichia coli (Gram-negative bacteria), and Candida albicans (fungus) to determine the antimicrobial activities. MTT assay was applied to MCF-7 (human breast cancer cell line) as well as on HCT-116 (human colon cancer cell line) to calculate the IC50 of different plant extracts. The GC-MS analysis showed that only petroleum ether extract has an abundance of cyclohexane compounds including $46.5 \%$ methyl-cyclohexane. Significant antibacterial and antifungal actions against the tested strains were shown by the petroleum ether and chloroform extracts in antimicrobial assay. Antibacterial activity against $S$.
\end{abstract}

aureus (SA) and E. coli (EC) was exhibited by methanolic extract, whereas no effect was observed on B. subtilis (BS) and $C$. albicans (CA). In MTT assay, the petroleum ether extract showed the greatest cytotoxic activity against MCF-7 $(3.77 \mu \mathrm{g} / \mathrm{mL})$ and HCT-116 $(3.09 \mu \mathrm{g} / \mathrm{mL})$ cells. The extract of chloroform also displayed significant cytotoxic effect but only against MCF-7 with $\mathrm{IC}_{50}$ values of $12.7 \mu \mathrm{g} / \mathrm{mL}$. The present study showed that the $R$. officinalis petroleum ether extract contains significant antimicrobial and cytotoxic activities which can be accredited to the plentiful manifestation of methyl-cyclohexane, methylbenzene and other cyclohexane derivatives, and it may be used to develop new antimicrobial and anticancer drugs.

Keywords Rosmarinus officinalis, Antimicrobial Activity, Cytotoxicity, Medicinal Plant

\section{Introduction}

Due to a sudden rise in the number of contagious diseases and the development of antimicrobial resistance against current drugs, drug development studies are vital to discovering novel medicinal compounds [1]. Nowadays, 
therapeutic research focuses on medicinal plants due to their perceived effectiveness, lower side effects, and lower cost compared to that of synthetic drugs [2]. Several medicines are produced from plants, for instance quinine from the cinchona tree, aspirin is secreted from a willow tree, whereas, opium poppy provides vital medicine known as morphine [3]. Medicinal plants have shown to be beneficial in the function of various systems in the human body. For example, several plant extracts have strong antioxidant, anti-inflammatory, antimicrobial, antitumor, and immunostimulatory properties [4]. Hence, the search for new and effective medicinal plants from around the world has gained importance.

The Albaha region of Saudi Arabia, located in the southwest between Makkah and Aseer, is surrounded by forests and agricultural land; therefore, it is an ideal habitat to diverse flora, including several medicinal plants [5]. Rosmarinus officinalis (rosemary), which belongs to the Lamiaceae family, is a woody medicinal plant that originated from the Mediterranean region [6]. The medicinal uses of $R$. officinalis including treatment for gastrointestinal diseases, memory enhancement, antidepressant, and as an anti-inflammatory agent have been known for centuries [7-10]. In addition, $R$. officinalis extracts show a high antioxidant activity and can be used to increase the shelf life of perishable food items $[11,12]$.

The antimicrobial activity of $R$. officinalis extracts is also widely reported [11,13-15]. It has been found that the leaf extract of rosemary inhibits the growth of two of the main species, Shigella sonnei and E. coli [14]. The presence of different types of phenolic compounds in rosemary extracts makes it an effective antioxidant and imparts antimicrobial action against various microbes [11].

The cytotoxic and antitumor effects of rosemary extracts have been demonstrated in several studies $[13,16-$ 20]. It has been reported that rosemary has a high content of phenolic compounds which contribute to its antitumor activity [21]. Polyphenols are phytochemicals that primarily modulate cell growth and inhibit tumor development [22]. The most common polyphenols present in $R$. officinalis are phenolic acids (rosmarinic acid and caffeic acid), diosmin, and apigenin [16,23].

A literature survey indicated that no study had previously evaluated the antimicrobial and anticancer activities of $R$. officinalis growing in the Albaha region. In the present study, we aimed to identify the chemical constituents that are present in different extracts of $R$. officinalis using GC-MS analysis and evaluate the antimicrobial and anticancer activities of these extracts and compare their efficiencies.

\section{Materials and Methods}

\subsection{Chemicals}

Streptomycin, Muller-Hinton agar, penicillin, Dulbecco's modified Eagle's medium (DMEM), dimethyl sulfoxide (DMSO), glutamine, 3-(4,5-dimethylthiaxolyl-2)-2,5-diphenyltetrazolium

bromide (MTT), high-glucose medium fetal bovine serum (FBS), methanol, ethanol, petroleum ether and chloroform were purchased from Sigma chemicals Co., USA.

\subsection{Plant Materials}

Rosemary plants were gained from a local farmer located in Albaha, Saudi Arabia in March 2019. The botanical identification of the plant was authenticated by Dr. Haider, Department of Biology, Albaha University. The obtained specimen was deposited in the Botany Laboratory of the Biology department in Albaha University, Albaha, Saudi Arabia.

\subsubsection{Extraction of Crude Extract}

The fresh aerial parts of the rosemary plant (stem and leaves) were air dried at room temperature for approximately 15 days. Around $250 \mathrm{~g}$ of the dried material was powdered using grinder machine (Pulverizer HR-30B, USA) and soaked in $500 \mathrm{~mL}$ ethanol with shaking for three days. Then they were filtrated using filter paper (Whatman no1). The residues were air dried and extracted into various fractions by using solvents with increasing polarity: petroleum ether, chloroform, and methanol. All filtrates were concentrated using a rotary evaporator (IKA RV-10, Germany) and air dried to dryness [24].

\subsection{GC-MS Analysis}

A Perkin Elmer model Clarus $600 \mathrm{~T}$, together with single quadrapole mass spectrometer, was applied for GC-MS analysis [25], with certain modifications. The chromatographic column used was an Elite 5-MS column (30 $\mathrm{m} \times 0.25 \mathrm{~mm} \times 0.25 \mu \mathrm{m}$ film thickness). High-purity helium was used as the gas carrier, at a flow rate of 1 $\mathrm{mL} / \mathrm{min}$. The injector temperature was $280^{\circ} \mathrm{C}$ and the split ratio was 20:1. At the beginning of the procedure a $40^{\circ} \mathrm{C}$ temperature was applied for $1 \mathrm{~min}$, and subsequently raised to $150^{\circ} \mathrm{C}$ at $10^{\circ} \mathrm{C} \mathrm{min}-1$ for $1 \mathrm{~min}$, afterwards a further increase to $300^{\circ} \mathrm{C}$ at $10^{\circ} \mathrm{C}$ min for 1 min was applied. The injector volume for each sample extract was $1 \mu \mathrm{l}$. The temperature of ion source was $220^{\circ} \mathrm{C}$ whereas the inlet line temperature was at $240^{\circ} \mathrm{C}$. The sample was analyzed by applying a scan range between 40 to $600 \mathrm{~m} / \mathrm{z}$ at electron energy of $70 \mathrm{eV}$, and the solvent delay of 4 min. Finally, NIST 2005 (National Institute of Standard and Technology library) and Wiley 2006 library were utilized to identify unknown compounds as previously described by Mosbah et. al. [26]. 


\subsection{Antimicrobial Activity Screening}

Four different extracts (ethanol, petroleum ether, chloroform, and methanol) of rosemary were tested against specific strains of American type culture collection (ATCC) included gram-positive bacteria: Staphylococcus aureus 29213 and Bacillus subtilis 6633, gram-negative bacteria: Escherichia coli 35218, and the fungus: Candida albicans 76615; the strains were obtained from the Microbiology Laboratory of King Abdulaziz University Hospital, Jeddah, Saudi Arabia.

Agar diffusion technique was conducted for the fundamental screening of the antimicrobial activity [27]. Briefly, petri dishes with $90 \mathrm{~mm}$ diameter were filled with $25 \mathrm{~mL}$ of Muller-Hinton agar containing $1 \mathrm{~mL}$ culture $(1 \times$ $106 \mathrm{CFU} / \mathrm{mL}$ ) of each strain. A sterile borer was used to create wells with diameter of $4 \mathrm{~mm}$ in seeded agar plates. Afterwards, the wells were permeated with $50 \mu \mathrm{L}$ of each of the rosemary extract $(10 \mathrm{mg} / \mathrm{mL})$ while DMSO of $10 \%$ was used as a negative control. Then the plates were nurtured at $37^{\circ} \mathrm{C}$ for $24 \mathrm{~h}$. The inhibitory effect was determined as the non-appearance of microbial growth in the field around the wells.

Independent experiments were performed against each of the tested microorganisms in triplicate. After incubation, a caliper was used to measure the broadness of the appearing growth inhibition zones. Subsequently, with the obtained measurements the average diameter was calculated and the mean values were accordingly tabulated.

\subsection{Cell Lines and Culture Medium}

Human breast cancer cell line (MCF-7) and colorectal cancer cell line (HCT-116) were provided by Dr. Thikryat, Pharmacology and Toxicology Laboratory, Faculty of Medicine, King Abdulaziz University, Jeddah, Saudi Arabia. The cells were cultured in $75 \mathrm{~cm}^{2}$ flasks of DMEM/high-glucose medium supplemented with $10 \%$ (v/v) FBS, 10,000 units/mL penicillin/streptomycin, and $1 \%(\mathrm{v} / \mathrm{v})$ glutamine and kept under a temperature of $37^{\circ} \mathrm{C}$ in a humidified incubator of $5 \% \mathrm{CO}_{2}$ as described by Cheung et. al. [20].

\subsubsection{Cytotoxicity Assay}

To indicate the cytotoxic activity of the rosemary extracts, the MTT colorimetric assay was observed [28]. MCF-7 and HCT-116 cells $\left(1 \times 10^{5}\right.$ cells $\left./ \mathrm{mL}\right)$ were implanted in 96-well plates in triplicate while they were incubated whole night at $37^{\circ} \mathrm{C}$ in a humidified incubator where $\mathrm{CO}_{2}$ percentage was $5 \%$ as previously described [29] with certain modifications. Rosemary extracts (ethanol, petroleum ether, chloroform, and methanol) at seven concentrations $\quad(10,25, \quad 50, \quad 100, \quad 250, \quad 500$, and $1000 \mu \mathrm{g} / \mathrm{mL}$ ) were added to the cells in triplicate and were further incubated at $37^{\circ} \mathrm{C}$ in a $\mathrm{CO}_{2}$ concentration of $5 \%$ for $72 \mathrm{~h}$. The extracts were dissolved in $0.1 \%$ DMSO as a vehicle. Appropriate control wells using untreated and DMSO-treated cells were prepared at the same time. As a reference drug (positive control), Paclitaxel was used. Subsequently, the medium of each well of all plates was detached and replaced with $100 \mu \mathrm{L}$ of complete medium (FBS and antibiotic) with $10 \%$ of MTT $(10 \mathrm{mg} / \mathrm{mL})$. These plates were incubated at $37^{\circ} \mathrm{C}$ in $5 \% \mathrm{CO}_{2}$ for $4 \mathrm{~h}$. The supernatants were removed after incubation and 100 $\mu \mathrm{L}$ of DMSO was combined to dissolve the purple formazan crystals obtained by the applicable cells. These cells were incubated for another $5 \mathrm{~min}$ at $37^{\circ} \mathrm{C}$ in $5 \% \mathrm{CO}_{2}$. The dissolved solutions at $570 \mathrm{~nm}$ were measured by using the SpectraMax M3 plate reader (Molecular Devices, San Jose, CA, USA). The cell viability was calculated using the following formula:

Cell viability $(\%)=(\mathrm{A}$ of treated cells $/ \mathrm{A}$ of control cells) $\times 100$, where $A=$ absorbance at $570 \mathrm{~nm}$.

\subsection{Statistical Analysis}

Data is expressed as a mean of three replicates \pm standard deviation. The half-maximal inhibitory concentration $\left(\mathrm{IC}_{50}\right)$ was estimated using the ED50 GraphPad Prism software 5.0 (GraphPad Software, Inc., CA, USA).

\section{Results}

\subsection{GC-MS Analysis}

Tables 1-4 list the chemical compositions of the four $R$. officinalis extracts. GC-MS analysis showed that the ethanolic extract contained 3,7-dimethyl-2,6-octadien-1-ol (22.59\%), bicyclo [3.1.1] hept-3-en-2-one (18.94\%), 1,8-cineole $(14.64 \%)$, carnosol $(10.95 \%), \quad$ and 2-(5-tert-butyl-4-hydroxy-2-methylphenyl) benzoic acid (7.39 \%); the petroleum ether extract contained methyl-cyclohexane (46.51\%), methyl-benzene (12.58\%), berbenone $(7.03 \%), \quad 1,8$-cineole $(6.11 \%), \quad$ and trans-geraniol (4.32\%); the chloroform extract contained bicyclo [3.1.1] hept-3-en-2-one (19.84\%), trans-geraniol (13.43\%), endo-borneol (7.96\%), 1,8-cineole (9.4\%), and vitamin E (5.24\%); the methanolic extract contained bicyclo [3.1.1] hept-3-en-2-one (14.82\%) caryophyllene diepoxide (10.5\%), 1,2,3-propanetriol (6.3\%), 5-(hydroxy)-2- furancarboxaldehyde (6.27\%), and borneol $(5.1 \%)$ is the most abundant components with a concentration higher than $5 \%$. 
Table 1. Chemical composition of Rosmarinus officinalis ethanolic extract

\begin{tabular}{|c|c|c|c|c|}
\hline & Name of Compound & $\mathbf{R T}^{\mathbf{a}}$ & Area \% & Area \\
\hline 1 & CIS-OCIMENE & 6.4 & 0.53 & 29825 \\
\hline 2 & 1,8-CINEOLE & 8.01 & 14.64 & 820450 \\
\hline 3 & ALPHA.-TERPINOLENE & 9 & 1.12 & 62921 \\
\hline 4 & CAMPHOR & 9.82 & 0.89 & 49615 \\
\hline 5 & 1,7 BICYCLO[2.2.1]HEPTAN-2-OL & 10.2 & 2.53 & 141634 \\
\hline 6 & 2-BICYCLO[3.1.1]HEPTAN-3-ONE & 10.26 & 0.32 & 18157 \\
\hline 7 & 4-TERPINEOL & 10.3 & 0.3 & 16880 \\
\hline 8 & 3-CYCLOHEXENE-1-METHANOL & 10.52 & 1.25 & 70094 \\
\hline 9 & 6,6-DIMETHYL-BICYCLO[3.1.1] HEPT-2-ENE-2-ETHANOL & 10.62 & 0.37 & 20545 \\
\hline 10 & BICYCLO[3.1.1]HEPT-3-EN-2-ONE & 10.73 & 18.94 & 1061125 \\
\hline 11 & 2-HYDROXY-2-METHYL-BUT-3-ENYL 2-METHYL-2(Z)-BUTENOATE & 10.81 & 1.16 & 65070 \\
\hline 12 & BETA.-CITRONELLOL & 10.88 & 0.53 & 29453 \\
\hline 13 & 3,7-DIMETHYL-2,6-OCTADIEN-1-OL & 11.23 & 22.59 & 1265704 \\
\hline 14 & (R-1,C-4)-P-MENTH-8-EN-1-OL & 11.3 & 0.31 & 17360 \\
\hline 15 & BORNYL ACETATE & 11.77 & 1.7 & 95437 \\
\hline 16 & TRANS-CARYOPHYLLENE & 13.96 & 0.52 & 29405 \\
\hline 17 & FORMIC ACID & 14.06 & 0.37 & 20907 \\
\hline 18 & PHENYLMETHYL ESTER OF (-)-CARYOPHYLLENE OXIDE & 16.31 & 0.27 & 15353 \\
\hline 19 & HEXADECANOIC ACID & 20.43 & 0.29 & 16240 \\
\hline 20 & 3,7,11,15-TETRAMETHYL 2-HEXADECEN-1-OL & 21.9 & 0.48 & 26712 \\
\hline 21 & 6(E),9(Z),13(E)-PENDECTRIENE & 22.14 & 0.53 & 29433 \\
\hline 22 & 11,14,17-EICOSATRIENOIC ACID & 22.4 & 0.23 & 12755 \\
\hline 23 & (5.BETA)-CHOL-7-ENE-12,24-DIOL & 23.07 & 0.3 & 17013 \\
\hline 24 & (+)-.BETA.-COSTOL & 23.84 & 0.3 & 16788 \\
\hline 25 & FERRUGINOL & 23.94 & 0.5 & 28110 \\
\hline 26 & $\begin{array}{c}\text { 3,8-DIHYDROXY-1,4,6-TRIMETHYL DIBENZO[B, } \\
\text { E][1,4]DIOXEPIN-11-ONE }\end{array}$ & 24.87 & 3.2 & 179414 \\
\hline 27 & ISOCARNOSOL & 25.11 & 0.5 & 27850 \\
\hline 28 & CARNOSOL & 25.27 & 10.95 & 613529 \\
\hline 29 & ISOCARNOSOL & 25.46 & 0.25 & 14249 \\
\hline 30 & FERRUGINOL & 25.54 & 0.69 & 38464 \\
\hline 31 & 2-(5-TERT-BUTYL-4-HYDROXY-2-METHYLPHENYL) BENZOIC ACID & 26.02 & 7.39 & 414265 \\
\hline 32 & 2-ALLYL-1,4-DIMETHOXY-6-METHYL BENZENE & 26.11 & 2.8 & 156808 \\
\hline 33 & CARNOSOL & 26.4 & 0.6 & 33474 \\
\hline 34 & (-)-ALPHA-COSTOL & 27.05 & 0.99 & 55377 \\
\hline 35 & 3,7,11-TRIDECATRIENENITRILE & 27.52 & 0.83 & 46385 \\
\hline 36 & DOCOSANE & 28.1 & 0.59 & 32789 \\
\hline 37 & SPINASTERONE & 28.52 & 0.25 & 13831 \\
\hline
\end{tabular}

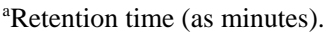


Table 2. Chemical composition of Rosmarinus officinalis petroleum ether extract

\begin{tabular}{|c|c|c|c|c|}
\hline & Name of Compound & $\mathbf{R T}^{\mathbf{a}}$ & Area \% & Area \\
\hline 1 & 2,2,4-TRIMETHYL-PENTANE & 3.23 & 0.26 & 67760 \\
\hline 2 & METHYL-CYCLOHEXANE & 3.33 & 46.51 & 12081537 \\
\hline 3 & ETHYL-CYCLOPENTANE & 3.43 & 0.78 & 202159 \\
\hline 4 & CYCLOPENTANE & 3.51 & 0.39 & 100798 \\
\hline 5 & 1,2,4-TRIMETHYL-TETRAHYDRO GERANIOL & 3.6 & 0.2 & 52528 \\
\hline 6 & 2,3-DIMETHYL-HEXANE & 3.72 & 0.2 & 52658 \\
\hline 7 & METHYL-BENZENE & 3.83 & 12.58 & 3267939 \\
\hline 8 & 3-METHYL-HEPTANE & 3.9 & 0.51 & 132387 \\
\hline 9 & 1,4-DIMETHYL-CYCLOHEXANE & 4.03 & 1.75 & 454445 \\
\hline 10 & METHYL-CYCLOHEPTANE & 4.15 & 0.16 & 42265 \\
\hline 11 & 2,4-DIMETHYL-HEPTANE & 4.27 & 1.87 & 485756 \\
\hline 12 & 1,2-DIMETHYL-CYCLOHEXANE & 4.32 & 0.21 & 55091 \\
\hline 13 & 1,3-DIMETHYL-CYCLOHEXANE & 4.41 & 0.11 & 29116 \\
\hline 14 & 2,6-DIMETHYL-HEPTANE & 4.7 & 0.15 & 39763 \\
\hline 15 & ETHYL- CYCLOHEXANE & 4.84 & 0.2 & 53235 \\
\hline 16 & 1,2-DIMETHYL-BENZENE & 5.37 & 0.56 & 146510 \\
\hline 17 & ALPHA.-PINENE & 6.43 & 0.28 & 71872 \\
\hline 18 & VERBENENE & 6.76 & 0.27 & 70230 \\
\hline 19 & 1,8-CINEOLE & 8.05 & 6.11 & 1587119 \\
\hline 20 & LINALOOL & 9.04 & 0.59 & 153253 \\
\hline 21 & 2,6-DIMETHYL-3,5-HEPTADIEN-2-OL & 9.43 & 0.11 & 29111 \\
\hline 22 & (.+-.)-CAMPHOR & 9.86 & 0.44 & 115010 \\
\hline 23 & PINOCARVONE & 10.07 & 0.11 & 27581 \\
\hline 24 & DELTA.-TERPINEOL & 10.18 & 0.14 & 35744 \\
\hline 25 & BORNEOL & 10.24 & 1.95 & 505392 \\
\hline 26 & BICYCLO[3.1.1]HEPTAN-3-ONE & 10.29 & 0.15 & 37908 \\
\hline 27 & 4-METHYL-1-3-CYCLOHEXEN-1-OL & 10.34 & 0.3 & 78997 \\
\hline 28 & 3-CYCLOHEXENE-1-METHANOL & 10.56 & 0.9 & 234079 \\
\hline 29 & BICYCLO[3.1.1]HEPT-2-ENE-2-ETHANOL & 10.66 & 0.22 & 57693 \\
\hline 30 & BERBENONE & 10.77 & 7.03 & 1827293 \\
\hline 31 & 4-METHYL-3-PENTEN-2-ONE & 10.85 & 0.4 & 105119 \\
\hline 32 & 3,7-DIMETHYL-6-OCTEN-1-OL & 10.91 & 0.12 & 30172 \\
\hline 33 & TRANS-GERANIOL & 11.27 & 4.32 & 1123131 \\
\hline 34 & 1-VINYL-1-(4-METHYL)PENTAN-3-ENE & 11.33 & 0.08 & 19854 \\
\hline 35 & ACETA-Z-CRYSANTHENYL & 11.73 & 0.19 & 48091 \\
\hline 36 & 1,7,7-TRIMETHYL-BICYCLO[2.2.1]HEPT-2-YL ESTER ACETIC ACID & 11.81 & 1.58 & 411378 \\
\hline 37 & TRANS-FARNESOL & 11.92 & 0.43 & 112082 \\
\hline 38 & NERYL ACETATE & 13.1 & 0.22 & 57353 \\
\hline 39 & 1,2-DIMETHOXY-4-(2-PRBENZENE & 13.48 & 0.07 & 17646 \\
\hline 40 & TRANS-CARYOPHYLLENE & 14 & 0.26 & 67407 \\
\hline 41 & CARYOPHYLLENE OXIDE & 16.33 & 0.05 & 13733 \\
\hline 42 & N-HEXADECANOIC ACID & 20.46 & 0.08 & 21366 \\
\hline 43 & 9-OCTADECENOIC ACID & 20.88 & 0.1 & 26928 \\
\hline 44 & 3,7,11,15-TETRAMETHYL 2-HEXADECEN-1-OL & 21.91 & 0.3 & 79085 \\
\hline 45 & OXIRANEMETHANOL & 22.16 & 0.17 & 44454 \\
\hline 46 & (-)-OBTUSANE & 23.6 & 0.04 & 10951 \\
\hline 47 & (-)-CARYOPHYLLENE OXIDE & 23.84 & 0.04 & 11129 \\
\hline 48 & FERRUGINOL & 23.95 & 0.28 & 73522 \\
\hline 49 & VITAMIN E & 24.35 & 0.62 & 161554 \\
\hline 50 & 1-(1,2-DICYANOETHENYL)[6](1,4)-NAPHTHALENOPHAN -1,2-CYCLOPROPANE & 24.6 & 0.87 & 226226 \\
\hline 51 & $\begin{array}{l}\text { 4,4-DIMETHYL-2-\{(Z)-[2-(N-P-TOLYLAMINO)- } \\
\text { 4-METHYL]PENT-1-ENYL\}-2-OXAZOLINE }\end{array}$ & 24.88 & 0.39 & 101217 \\
\hline 52 & 2-HYDROXY-1 OCTADECANOIC ACID & 25.28 & 1.19 & 310016 \\
\hline 53 & 13-ISOPROPYLPODOCARPEN-12-OL-20 AL & 25.54 & 0.12 & 30868 \\
\hline 54 & 2-ALLYL-1,4-DIMETHOXY-6-METHYL BENZENE & 26.11 & 0.48 & 123703 \\
\hline 55 & TRITETRACONTANE & 26.69 & 0.4 & 104941 \\
\hline 56 & TETRATETRACONTANE & 28.08 & 1.88 & 487837 \\
\hline
\end{tabular}

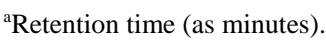



of Rosmarinus officinalis (Rosemary): A Comparative Study

Table 3. Chemical composition of Rosmarinus officinalis chloroform extract

\begin{tabular}{|c|c|c|c|c|}
\hline & Name of Compound & $\mathbf{R T}^{\mathbf{a}}$ & Area $\%$ & Area \\
\hline 1 & VERBENENE & 6.76 & 0.78 & 34849 \\
\hline 2 & METHYL(1-METHYLETHYL)-BENZENE & 7.9 & 0.67 & 29954 \\
\hline 3 & 1,8-CINEOLE & 8.04 & 9.4 & 418656 \\
\hline 4 & LINALOOL & 9.04 & 2.14 & 95457 \\
\hline 5 & CHRYSANTHENONE & 9.43 & 0.34 & 15123 \\
\hline 6 & CAMPHOR & 9.86 & 1.17 & 52075 \\
\hline 7 & BICYCLO[2.2.1]HEPTAN-3-ONE & 10.07 & 0.34 & 15052 \\
\hline 8 & 3-CYCLOHEXENE-1-METHANOL & 10.17 & 0.68 & 30251 \\
\hline 9 & ENDO-BORNEOL & 10.23 & 7.96 & 354651 \\
\hline 10 & PINOCAMPHONE & 10.29 & 0.6 & 26773 \\
\hline 11 & 4-TERPINEOL & 10.34 & 1.24 & 55258 \\
\hline 12 & LINALYL PROPIONATE & 10.55 & 3.25 & 144673 \\
\hline 13 & B6,6-DIMETHYLICYCLO[3.1.1] HEPT-2-ENE-2-ETHANOL & 10.65 & 1.14 & 50601 \\
\hline 14 & BICYCLO[3.1.1]HEPT-3-EN-2-ONE & 10.76 & 19.84 & 883454 \\
\hline 15 & 2-HYDROXY-2-METHYL-BUT-3-ENYL & 10.84 & 1.21 & 53862 \\
\hline 16 & 3,7-DIMETHYL-6-OCTEN-1-OL & 10.91 & 0.48 & 21453 \\
\hline 17 & TRANS-GERANIOL & 11.26 & 13.43 & 598086 \\
\hline 18 & (+)-TRANS-CARAN-TRANS-2-OL & 11.33 & 0.27 & 11908 \\
\hline 19 & 1,7,7-TRIMETHYL-BICYCLO[2.2.1] HEPT-2-YL ESTER ACETIC ACID & 11.81 & 2.16 & 96168 \\
\hline 20 & NEROLIC ACID & 12.61 & 0.81 & 36187 \\
\hline 21 & NERYL ACETATE & 13.09 & 0.67 & 29892 \\
\hline 22 & (-)-CARYOPHYLLENE OXIDE & 16.33 & 0.49 & 21870 \\
\hline 23 & TETRADECANAL & 16.55 & 0.19 & 8578 \\
\hline 24 & (+)-.BETA.-COSTOL & 17.24 & 0.37 & 16650 \\
\hline 25 & GLOBULOL & 17.41 & 0.12 & 5507 \\
\hline 26 & NEOPHYTADIENE & 19.65 & 0.24 & 10760 \\
\hline 27 & XYCAINE & 19.81 & 1.16 & 51518 \\
\hline 28 & HEXADECANOIC ACID & 20.45 & 0.73 & 32616 \\
\hline 29 & 1-TETRADECENE & 20.87 & 0.34 & 14987 \\
\hline 30 & 3,7,11,15-TETRAMETHYL 2-HEXADECEN-1-OL & 21.9 & 0.89 & 39725 \\
\hline 31 & 3.BETA ERGOSTA-7,22-DIEN-3-OL & 23.1 & 0.19 & 8541 \\
\hline 32 & TETRACOSANE & 23.37 & 3.15 & 140177 \\
\hline 33 & 12-CHLOROMERCURIOTOTARA-8,11,13-TRIEN-13-OL & 23.95 & 0.78 & 34715 \\
\hline 34 & 9-OCTADECENAMIDE & 24.1 & 1.18 & 52608 \\
\hline 35 & ISOCARNOSOL & 24.27 & 0.51 & 22532 \\
\hline 36 & VITAMIN E & 24.35 & 5.24 & 233497 \\
\hline 37 & CARNOSOL & 25.26 & 4.13 & 184040 \\
\hline 38 & 5.ALPHA.STIGMAST-24(28)-ENE & 26.1 & 2.72 & 120907 \\
\hline 39 & PENTATRIACONTANE & 27.82 & 2.92 & 130105 \\
\hline 40 & TRITETRACONTANE & 28.07 & 4.95 & 220319 \\
\hline 41 & (-)-ALLOAROMADENDRONE & 28.51 & 0.46 & 20356 \\
\hline
\end{tabular}

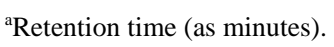


Table 4. Chemical composition of Rosmarinus officinalis methanolic extract

\begin{tabular}{|c|c|c|c|c|}
\hline & Name of Compound & $\mathbf{R T}^{\mathbf{a}}$ & Area \% & Area \\
\hline 1 & 2-FURANMETHANOL & 5.11 & 0.34 & 6066 \\
\hline 2 & DL-GLYCERALDEHYDE DIMER & 5.73 & 3.79 & 68546 \\
\hline 3 & 2-HYDROXY-2-CYCLOPENTEN-1-ONE & 6.22 & 0.42 & 7615 \\
\hline 4 & 2,4-DIHYDROXY-2,5-DIMETHYL-3(2H)-FURAN-3-ONE & 7.15 & 1.02 & 18436 \\
\hline 5 & 1-BUTOXY-2-PROPANOL ACETATE & 7.29 & 1.85 & 33377 \\
\hline 6 & 1,8-CINEOLE & 8.04 & 2.47 & 44618 \\
\hline 7 & HYDROXY DIMETHYL FURANONE & 8.34 & 0.61 & 11051 \\
\hline 8 & 3-HYDROXY-2-METHYL-4H-PYRAN-4-ONE & 8.64 & 1.12 & 20207 \\
\hline 9 & 2-ACETYL-5-METHYLFURAN & 8.86 & 0.47 & 8425 \\
\hline 10 & 3-AMINO-2-OXAZOLIDINONE & 9.53 & 0.82 & 14881 \\
\hline 11 & CARYOPHYLLENE DIEPOXIDE & 9.73 & 10.52 & 190193 \\
\hline 12 & BORNEOL & 10.23 & 5.1 & 92248 \\
\hline 13 & Z-3-HEXENYL 2-METHYLPROPANOATE & 10.38 & 0.67 & 12076 \\
\hline 14 & 1,2-BENZENEDIOL & 10.47 & 3.62 & 65373 \\
\hline 15 & 3-CYCLOHEXENE-1-METHANOL & 10.54 & 1.3 & 23449 \\
\hline 16 & (-)-CARYOPHYLLENE OXIDE & 10.64 & 0.57 & 10257 \\
\hline 17 & BICYCLO[3.1.1]HEPT-3-EN-2-ONE & 10.76 & 14.82 & 267852 \\
\hline 18 & 5-(HYDROXY)-2-FURANCARBOXALDEHYDE & 10.88 & 6.27 & 113269 \\
\hline 19 & 1,2,3-PROPANETRIOL & 11.13 & 6.3 & 113800 \\
\hline 20 & 2,7-OCTADIENE-1,6-DIOL & 11.57 & 1.45 & 26172 \\
\hline 21 & 2,3-DIHYDRO-3-HYDROXY-4H-PYRAN-4-ONE & 11.69 & 2.61 & 47161 \\
\hline 22 & EXOBORNYL ACETATE & 11.81 & 0.96 & 17275 \\
\hline 23 & 4-ACETOXY-3-METHOXYSTYRENE & 12.16 & 1.55 & 28005 \\
\hline 24 & 2,6-DIMETHOXY-PHENOL & 12.66 & 0.84 & 15229 \\
\hline 25 & 4-ETHYL-1,3-BENZENEDIOL & 13.18 & 0.73 & 13144 \\
\hline 26 & 2-HYDROXY-6-METHYL BENZALDEHYDE & 14.1 & 10.64 & 192328 \\
\hline 27 & (Z)-NON-2-EN-6,8-DIYNOIC ACID & 14.58 & 0.24 & 4263 \\
\hline 28 & D-ALLOSE & 14.91 & 3.38 & 61092 \\
\hline 29 & 4-HYDROXY-3-METHYL-BENZOIC ACID & 15.79 & 0.58 & 10482 \\
\hline 30 & 3-DEOXY-D-MANNOIC LACTONE & 16.34 & 4.32 & 78104 \\
\hline 31 & DECANAL & 16.59 & 1.57 & 28325 \\
\hline 32 & GINGEROL & 16.96 & 0.55 & 9932 \\
\hline 33 & 4-((1E)-3-HYDROXY-1-PROPENYL)-2-METHOXYPHENOL & 18.1 & 0.79 & 14315 \\
\hline 34 & DL-3,4-DIMETHYL-3,4-HEXANEDIOL & 18.36 & 0.77 & 13843 \\
\hline 35 & 4-HYDROXYL-PROLINE & 19.83 & 1.39 & 25078 \\
\hline 36 & HEXADECANOIC ACID & 20.44 & 0.66 & 11964 \\
\hline
\end{tabular}

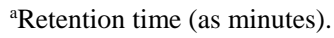

\subsection{Antimicrobial Activity}

The in vitro antibacterial and antifungal assays were performed using the ethanol, petroleum ether, chloroform, and methanol extracts obtained from $R$. officinalis. Results showed that chloroform, methanol, and petroleum ether extracts exhibited significant antimicrobial activity towards most of the selected strains (Table 5). The highest activities were observed for petroleum ether extract with zones of inhibition of $22 \mathrm{~mm}, 14 \mathrm{~mm}, 21 \mathrm{~mm}$, and $22 \mathrm{~mm}$, followed by the chloroform extract with zones of inhibition of $18 \mathrm{~mm}, 12 \mathrm{~mm}, 15 \mathrm{~mm}$, and $12 \mathrm{~mm}$ against SA, BS, EC, and CA strains, respectively. The methanol extract showed significant activity against SA with a zone of inhibition of $20 \mathrm{~mm}$ and a moderate activity against EC with a zone of inhibition of $7 \mathrm{~mm}$. Notably, the ethanol extract did not exhibit any activity against the tested strains. 

of Rosmarinus officinalis (Rosemary): A Comparative Study

Table 5. Antibacterial and antifungal activities of Rosmarinus officinalis extracts

\begin{tabular}{ccccc}
\hline & \multicolumn{4}{c}{ Zone diameter $^{\mathbf{a}}(\mathbf{m m})$} \\
\cline { 2 - 5 } Extracts & \multicolumn{2}{c}{ Gram-positive bacteria } & Gram-negative bacteria & Fungus \\
\cline { 2 - 5 } & $\begin{array}{c}\text { Staphylococcus } \\
\text { aureus }\end{array}$ & $\begin{array}{c}\text { Bacillus } \\
\text { subtilis }\end{array}$ & Escherichia coli & Candida albicans \\
\hline Chloroform & $18 \pm 0.05$ & $12 \pm 0.07$ & $15 \pm 0.04$ & $12 \pm 0.04$ \\
\hline Methanol & $20 \pm 0.04$ & - & $7 \pm 0.12$ & - \\
\hline Ethanol & - & - & - & $22 \pm 0.05$ \\
\hline Petroleum ether & $22 \pm 0.04$ & $14 \pm 0.14$ & & - \\
\hline
\end{tabular}

\subsection{Cytotoxic Activity}

To discover new agents capable of hindering the propagation of human breast cancer as well as human colorectal cancer cell lines, different extracts (ethanol, petroleum ether, chloroform, and methanol) of rosemary were tested at seven concentrations against MCF-7 and HCT-116 cells. The IC50 values of the extracts were determined to evaluate their effectiveness. As shown in Table 6, the IC50 for MCF-7 ranged from 3.77 to 76.2 $\mu \mathrm{g} / \mathrm{mL}$, whereas the IC50 for HCT-116 ranged from 3.09 to $324 \mu \mathrm{g} / \mathrm{mL}$. Petroleum ether extracts showed the greatest cytotoxic effect against MCF-7 and HCT-116 cells with IC50 values of 3.77 and $3.09 \mu \mathrm{g} / \mathrm{mL}$, respectively. Chloroform as well as methanol extracts also displayed significant cytotoxic effects against MCF-7 cells with IC50 values of 12.7 and $23.59 \mu \mathrm{g} / \mathrm{mL}$, respectively. In contrast, ethanol extract displayed a moderate cytotoxic effect with an IC50 of $76.2 \mu \mathrm{g} / \mathrm{mL}$ against MCF-7. The chloroform extract showed a moderate cytotoxic effect against HCT-116 cells with an IC50 of $59.1 \mu \mathrm{g} / \mathrm{mL}$, whereas methanol as well as ethanol extracts exhibited weak cytotoxic effects against HCT-116 cells with IC50 values of 259 and $324 \mu \mathrm{g} / \mathrm{mL}$, respectively.

Table 6. The $\mathrm{IC}_{50}$ of Rosmarinus officinalis extracts against tested human cancer cell lines

\begin{tabular}{|c|c|c|}
\hline Compound & $\begin{array}{c}\mathbf{I C}_{\mathbf{5 0}}{ }^{*}(\boldsymbol{\mu g} / \mathbf{m L}) \\
\mathbf{M C F}-7\end{array}$ & $\begin{array}{c}\mathbf{I C}_{\mathbf{5 0}}{ }^{*}(\boldsymbol{\mu g} / \mathbf{m L}) \\
\text { HCT-116 }\end{array}$ \\
\hline Chloroform extract & $12.7 \pm 5.4 \times 10^{-6}$ & $59.1 \pm 1.8 \times 10^{-6}$ \\
\hline Methanol extract & $23.59 \pm 0.27 \times 10^{-6}$ & $259 \pm 4.7 \times 10^{-5}$ \\
\hline Ethanol extract & $76.2 \pm 2.2 \times 10^{-5}$ & $324 \pm 3.37 \times 10^{-5}$ \\
\hline $\begin{array}{c}\text { Petroleum ether } \\
\text { extract }\end{array}$ & $3.77 \pm 1.3 \times 10^{-6}$ & $3.09 \pm 2.28 \times 10^{-6}$ \\
\hline $\begin{array}{c}\text { Standard drug } \\
\text { (paclitaxel) }\end{array}$ & $0.23 \pm 2.2 \times 10^{-6}$ & $0.32 \pm 5.7 \times 10^{-6}$ \\
\hline
\end{tabular}

\section{Discussion}

GC-MS was performed to investigate the phytochemical composition of each rosemary extract. The petroleum ether extract contained the most constituents (56 compounds). Approximately 39 of these constituents are unique, including the bioactive compounds methyl-cyclohexane (46.5\%), methylbenzene (12.58\%), and alpha-pinene $(0.28 \%)$, which are not found in other $R$. officinalis extracts of this study.

The common bioactive compound that was determined in all extracts was 1,8-cineole. Terpinol and camphor were found in ethanol, petroleum ether, and chloroform extracts, excluding the methanolic extract. The (-)-caryophyllene oxide and borneol were found in methanol, petroleum ether, and chloroform extracts, but not in the ethanolic extract. Geraniol and vitamin E were found in the petroleum ether and chloroform extracts. Few differences were observed in the ingredients and combinations of the extracts in comparison to those which were mentioned before in $R$. officinalis collected from various geological areas, which may be credited to certain elements as follows: climate, time of collection, and mode of extraction $[30,31]$.

The microorganisms tested are morphologically and physiologically different. Petroleum ether extracts exhibited a great antimicrobial activity against all tested strains, followed by chloroform extracts. The strong antimicrobial effect of petroleum ether and chloroform extracts can be attributed to the presence of the phytoconstituents geraniol and borneol that show antimicrobial action against various species [32,33]. Our findings are consistent with various studies that highlight the antibacterial and antifungal actions of extracts obtained from Rosmarinus species. For instance, oil extracts from $R$. officinalis have displayed how to prohibit the development of bacteria such as EC, Listeria monocytogenes, and SA [34]. In addition, it has been reported that $R$. officinalis oil extract inhibits the activity of CA by preventing the adhesion of this fungus via denaturation of the cellular structures and alteration of membrane permeability [35].

The cytotoxic of $R$. officinalis' extracts impacts on MCF-7 and HCT-116 cell lines were investigated by performing the MTT assay. The petroleum ether extract demonstrated the greatest cytotoxic effect against MCF-7 and HCT-116 cells with $\mathrm{IC}_{50}$ values of 3.77 and 3.09 $\mu \mathrm{g} / \mathrm{mL}$, respectively. Chloroform and methanol extracts exhibited significant cytotoxic effects against MCF-7 cells with $\mathrm{IC}_{50}$ values of 12.7 and $23.59 \mu \mathrm{g} / \mathrm{mL}$, respectively, but not against HCT-116 cells. The National Cancer 
Institute (USA) plant screening program states an $\mathrm{IC}_{50}$ of $<20 \mu \mathrm{g} / \mathrm{mL}$ as a threshold for an extract to be considered as anticancer, following an incubation period of 48 and $72 \mathrm{~h}$ [36,37]. The findings of the current study are consistent with previous anticancer studies that report the potent cytotoxic effects of $R$. officinalis extracts on colon cancer cell lines [38]. Similarly, previous studies have demonstrated a significant cytotoxic effect of $R$. officinalis extract on breast cancer cell lines [39].

Remarkably, the petroleum ether extract showed the highest antimicrobial and anticancer activities compared to those of other $R$. officinalis extracts. This may be associated with the existence of a high number of methyl-cyclohexane, methylbenzene, and other cyclohexane derivatives. Cyclohexane and its practically alternative derivatives are vital compounds showing distinct biological actions involving antioxidant, anticancer, cytotoxic, analgesic, anti-inflammatory, and antithrombin activities [40-46].

\section{Conclusions}

Various cancers and infectious diseases contribute to morbidity and mortality worldwide. This situation requires the development of new cost-effective therapies that are more effective than the present therapies. In this aspect, medicinal plants are a useful source of novel therapeutic substances, and several drugs are composed of plant-derived chemicals. The current study was carried out to analyze the phytochemical contents of different extracts of $R$. officinalis obtained from the Albaha region and evaluate the antimicrobial and anticancer properties of each extract. The petroleum ether extract demonstrated significant antimicrobial and anticancer activities. This may be linked to the presence of a high amount of methyl-cyclohexane, methylbenzene, and other cyclohexane derivatives that are already in consideration to display antimicrobial and anticancer activities. Advanced research is important to evaluate which components are mainly responsible for these antimicrobial and anticancer activities and their mechanism of action.

\section{Acknowledgements}

The principal investigator of this study acknowledges the Deanship of Scientific Research, Albaha University, Albaha, KSA for financial support (grant \#17-1439).

\section{REFERENCES}

[1] Vnutskikh ZA, Shklyaev Y V, Odegova TF, Chekryshkin YS, Tolstikov AG, El'chishcheva N V, et al. Synthesis and antimicrobial activity of mono-and biquaternized derivatives of dipyridylethanes and dipyridylethylenes. Pharm Chem J 2006;40(4):194-198.

[2] Firenzuoli F, Gori L. Herbal medicine today: clinical and research issues. Evidence-Based Complement Altern Med $2007 ; 4$.

[3] Karunamoorthi K, Jegajeevanram K, Vijayalakshmi J, Mengistie E. Traditional medicinal plants: a source of phytotherapeutic modality in resource-constrained health care settings. J Evid Based Complementary Altern Med 2013;18(1):67-74.

[4] Ekor M. The growing use of herbal medicines: issues relating to adverse reactions and challenges in monitoring safety. Front Pharmacol 2014;4:177.

[5] Ali NAA, Al Sokari SS, Gushash A, Anwar S, Al-Karani K, Al-Khulaidi A. Ethnopharmacological survey of medicinal plants in Albaha Region, Saudi Arabia. Pharmacognosy Res 2017;9(4):401.

[6] Rotblatt M. Herbal medicine: expanded commission E monographs. Ann Intern Med 2000;133(6):487.

[7] Hamidpour R, Hamidpour S, Elias G. Rosmarinus officinalis (Rosemary): a novel therapeutic agent for antioxidant, antimicrobial, anticancer, antidiabetic, antidepressant, neuroprotective, anti-inflammatory, and anti-obesity treatment. Biomed J Sci Tech Res 2017;1(4):16.

[8] Habtemariam S. The therapeutic potential of rosemary (Rosmarinus officinalis) diterpenes for Alzheimer's disease. Evidence-Based Complement Altern Med 2016;2016.

[9] Naimi M, Vlavcheski F, Shamshoum H, Tsiani E. Rosemary extract as a potential anti-hyperglycemic agent: current evidence and future perspectives. Nutrients 2017;9(9):968

[10] De Oliveira JR, Camargo SEA, De Oliveira LD. Rosmarinus officinalis L.(rosemary) as therapeutic and prophylactic agent. J Biomed Sci 2019;26(1):5.

[11] Nieto G, Ros G, Castillo J. Antioxidant and antimicrobial properties of rosemary (Rosmarinus officinalis, L.): A Review. Medicines 2018;5(3):98.

[12] Rašković A, Milanović I, Pavlović N, Ćebović T, Vukmirović S, Mikov M. Antioxidant activity of rosemary (Rosmarinus officinalis L.) essential oil and its hepatoprotective potential. BMC Complement Altern Med 2014;14(1):225.

[13] Wang W, Li N, Luo M, Zu Y, Efferth T. Antibacterial activity and anticancer activity of Rosmarinus officinalis $\mathrm{L}$. essential oil compared to that of its main components. Molecules 2012;17(3):2704-2713.

[14] Bozin B, Mimica-Dukic N, Samojlik I, Jovin E. Antimicrobial and antioxidant properties of rosemary and sage (Rosmarinus officinalis L. and Salvia officinalis L., Lamiaceae) essential oils. $J$ Agric Food Chem 2007;55(19):7879-7885.

[15] Tavassoli SK, Mousavi SM, Emam-Djomeh Z, Razavi SH Chemical composition and evaluation of antimicrobial properties of Rosmarinus officinalis L. essential oil. African J Biotechnol 2011;10(63):13895-13899.

[16] Yesil-Celiktas O, Sevimli C, Bedir E, Vardar-Sukan F. 
Inhibitory effects of rosemary extracts, carnosic acid and rosmarinic acid on the growth of various human cancer cell lines. Plant Foods Hum Nutr 2010;65(2):158-163.

[17] Moore J, Yousef M, Tsiani E. Anticancer effects of rosemary (Rosmarinus officinalis L.) extract and rosemary extract polyphenols. Nutrients 2016;8(11):731.

[18] González-Vallinas M, Reglero G, Ramírez de Molina A. Rosemary (Rosmarinus officinalis L.) extract as a potential complementary agent in anticancer therapy. Nutr Cancer 2015;67(8):1223-1231.

[19] H R. Rosmarinus officinalis (Rosemary): A Novel Therapeutic Agent for Antioxidant, Antimicrobial, Anticancer, Antidiabetic, Antidepressant, Neuroprotective, Anti-Inflammatory and Anti-Obesity Treatment. Herb Med Open Access 2017;03(02):1-6.

[20] Cheung S, Tai J. Anti-proliferative and antioxidant properties of rosemary Rosmarinus officinalis. Oncol Rep 2007;17(6):1525-1531.

[21] Moore J, Yousef M, Tsiani E. Anticancer effects of rosemary (Rosmarinus officinalis L.) extract and rosemary extract polyphenols. 2016 doi:10.3390/nu8110731.

[22] Kar S, Palit S, Ball WB, Das PK. Carnosic acid modulates $\mathrm{Akt} / \mathrm{IKK} / \mathrm{NF}-\mathrm{kB}$ signaling by PP2A and induces intrinsic and extrinsic pathway mediated apoptosis in human prostate carcinoma PC-3 cells. Apoptosis 2012;17(7):735747.

[23] Andrade JM, Faustino C, Garcia C, Ladeiras D, Reis CP, Rijo P. Rosmarinus officinalis L.: an update review of its phytochemistry and biological activity. Futur Sci OA 2018;4(4):FSO283.

[24] Jeyaseelan EC, Jenothiny S, Pathmanathan MK, Jeyadevan JP. Antibacterial activity of sequentially extracted organic solvent extracts of fruits, flowers and leaves of Lawsonia inermis L. from Jaffna. Asian Pac J Trop Biomed 2012;2(10):798-802.

[25] Al Hashmi LS, Hossain MA, Weli AM, Al-Riyami Q, AlSabahi JN. Gas chromatography-mass spectrometry analysis of different organic crude extracts from the local medicinal plant of Thymus vulgaris L. Asian Pac J Trop Biomed 2013;3(1):69-73.

[26] Mosbah H, Louati H, Boujbiha MA, Chahdoura H, Snoussi M, Flamini G, et al. Phytochemical characterization, antioxidant, antimicrobial and pharmacological activities of Feijoa sellowiana leaves growing in Tunisia. Ind Crops Prod 2018;112:521-531.

[27] Institute C and LS. Methods for dilution antimicrobial susceptibility tests for bacteria that grow aerobically. Approv Stand 2006;26:14-16.

[28] González ML, Joray MB, Laiolo J, Crespo MI, Palacios SM, Ruiz GM, et al. Cytotoxic activity of extracts from plants of central Argentina on sensitive and multidrug-resistant leukemia cells: isolation of an active principle from Gaillardia megapotamica. Evidence-Based Complement Altern Med 2018;2018.

[29] Mansour R Ben, Jilani IBH, Bouaziz M, Gargouri B, Elloumi N, Attia H, et al. Phenolic contents and antioxidant activity of ethanolic extract of Capparis spinosa. Cytotechnology 2016;68(1):135-142.
[30] Satyal P, Jones TH, Lopez EM, McFeeters RL, Ali NAA, Mansi I, et al. Chemotypic characterization and biological activity of Rosmarinus officinalis. Foods 2017;6(3):20.

[31] Diab Y, Auezova L, Chebib H, Chalchat J-C, Figueredo G. Chemical composition of Lebanese rosemary (Rosmarinus officinalis L.) essential oil as a function of the geographical region and the harvest time. J Essent Oil Res 2002;14(6):449-452.

[32] Tabanca N, Kırımer N, Demirci B, Demirci F, Başer KHC. Composition and antimicrobial activity of the essential oils of Micromeria cristata subsp. phrygia and the enantiomeric distribution of borneol. $J$ Agric Food Chem 2001;49(9):4300-4303.

[33] Lira MHP de, Andrade Júnior FP de, Moraes GFQ, Macena $\mathrm{G}$ da S, Pereira F de O, Lima IO. Antimicrobial activity of geraniol: An integrative review. J Essent Oil Res 2020;32(3):187-197.

[34] Rafie H, Soheila H, Grant E. Rosmarinus officinalis (Rosemary): A Novel Therapeutic Agent for Antioxidant, Antimicrobial, Anticancer, Antidiabetic, Antidepressant, Neuroprotective, Anti-Inflammatory and Anti-Obesity Treatment. J Herb Med 2017;3(2):8.

[35] Cavalcanti YW, Almeida L de FD de, Padilha WWN. Anti-adherent activity of Rosmarinus officinalis essential oil on Candida albicans: an SEM analysis. Rev Odonto Ciência 2011;26(2):139-144.

[36] Sufian AS, Ramasamy K, Ahmat N, Zakaria ZA, Yusof MIM. Isolation and identification of antibacterial and cytotoxic compounds from the leaves of Muntingia calabura L. J Ethnopharmacol 2013;146(1):198-204.

[37] Srisawat T, Chumkaew P, Heed-Chim W, Sukpondma Y, Kanokwiroon K. Phytochemical screening and cytotoxicity of crude extracts of Vatica diospyroides Symington Type LS. Trop J Pharm Res 2013;12(1):71-76.

[38] Yan M, Li G, Petiwala SM, Householter E, Johnson JJ. Standardized rosemary (Rosmarinus officinalis) extract induces $\mathrm{Nrf} 2 /$ sestrin-2 pathway in colon cancer cells. $J$ Funct Foods 2015;13:137-147.

[39] Yesil-Celiktas O, Sevimli C, Bedir E, Vardar-Sukan F. Inhibitory effects of rosemary extracts, carnosic acid and rosmarinic acid on the growth of various human cancer cell lines. Plant foods Hum Nutr 2010;65(2):158-163.

[40] Sharma M, Joshi P, Kumar N, Joshi S, Rohilla RK, Roy N, et al. Synthesis, antimicrobial activity and structure-activity relationship study of $\mathrm{N}, \mathrm{N}$-dibenzyl-cyclohexane-1, 2-diamine derivatives. Eur J Med Chem 2011;46(2):480487.

[41] Song L, Kang H, Liu D, Dai Z, He J, Wang B, et al. Dimedone \{2-[(4-hydroxy-phenylamino)-methylene]-5, 5-dimethyl-cyclohexane-1, 3-dione \} plays an important role in breast cancer treatment. Trop $J$ Pharm Res 2015;14(9):1719-1722.

[42] Modak B, Torres R, De Saint Pierre M, Katherine S, Armijo A, Caviedes R, et al. In vitro antiproliferative activity of 3 H-spiro [1-benzofuran-2, 1'-cyclohexane] derivatives. Boletín Latinoam y del Caribe Plantas Med y Aromáticas 2011;10(3):281-288. 
[43] Lallo S, Lee S, Dibwe DF, Tezuka Y, Morita H. A new polyoxygenated cyclohexane and other constituents from Kaempferia rotunda and their cytotoxic activity. Nat Prod Res 2014;28(20):1754-1759.

[44] Kumar D, Kumbukgolla W, Jayaweera S, Bailey M, Alling $\mathrm{T}$, Ollinger $\mathrm{J}$, et al. Antibacterial activity of adamantyl substituted cyclohexane diamine derivatives against methicillin resistant Staphylococcus aureus and Mycobacterium tuberculosis. RSC Adv 2014;4(23):1196211966.
[45] Amin KM, Kamel MM, Anwar MM, Khedr M, Syam YM. Synthesis, biological evaluation and molecular docking of novel series of spiro $[(2 \mathrm{H}, 3 \mathrm{H})$ quinazoline-2, 1 '-cyclohexan]-4 $(1 \mathrm{H})$-one derivatives as anti-inflammatory and analgesic agents. Eur J Med Chem 2010;45(6):21172131.

[46] Shoaib M, Aygun AI, Ganbarov K. Cyclohexane and its functionally substituted derivatives: Important class of organic compounds with potential antimicrobial activities. $J$ Microbiol Biotechnol Food Sci 2019;9(1):84. 